\title{
Investigations on specimen design and mounting for Split Hopkinson Tension Bar (SHTB) experiments
}

\author{
Noah Ledford ${ }^{1, \mathrm{a}}$, Hanna Paul ${ }^{1, \mathrm{~b}}$, Georg Ganzenmüller ${ }^{1, \mathrm{c}}$, Michael May ${ }^{1, \mathrm{~d}}$, Matthias Höfemann ${ }^{2, \mathrm{e}}$, Manuel Otto ${ }^{2, \mathrm{f}}$, \\ and Nikica Petrinic ${ }^{1,3, g}$ \\ ${ }^{1}$ Fraunhofer Institute for High-Speed Dynamics, Ernst-Mach-Institut, EMI, Eckerstr. 4, 79104 Freiburg, Germany \\ ${ }^{2}$ Salzgitter Mannesmann Forschung GmbH, Eisenhüttenstrasse 99, 38239 Salzgitter, Germany \\ ${ }^{3}$ Department of Engineering Science, University of Oxford, Parks Road, Oxford OX1 3PJ, UK
}

\begin{abstract}
Split Hopkinson Tension Bar (SHTB) experiments can be used to test the material behavior with high strain rates in tension loading. The influence of the specimen mounting and the specimen design on the test results was investigated. Three mounting methods were tested. The best signal is achieve using a mounting based on form fit. The three tested specimen designs all lead to a valid fracture behavior, but result in a different local strain rate.
\end{abstract}

\section{Introduction}

For various applications, material characterization with strain rates of $1000 \mathrm{~s}^{-1}$ and higher are of interest. Split Hopkinson Bar (SHB) testing methods are often used for this range of strain rates. In these experiments, a specimen is placed between two long elastic rods, the input and the output bar. The input bar is impacted with a projectile inducing a stress wave, which travels through the input bar into the specimen and into the output bar [1]. As opposed to the traditional Split Hopkinson Pressure Bar (SHPB) test, where the sample is simply placed between the input and output bars, a Split Hopkinson Tension Bar (SHTB) test requires a firm attachment of the sample to the bars, which is often difficult to realize. Another concern that arises with the SHTB is the tapering of the specimen from where it is attached to the input bar to the gauge section where within which the response of investigated materials is observed and quantified. Using a recent SHTB design derived from [2] which allows a longer loading pulse to be exerted along the loading bar with enhanced alignment by means of a lower pressure firing chamber, the effects of specimen design and mounting are investigated.

The challenge of attaching a specimen to the bars is addressed in different ways. One approach is to utilize an external thread on an axially symmetric specimen which engages an internal thread at the end of the bars. However, for materials without axial symmetry such as sheet metals or composites, other ways of mounting have to be employed. An alternative approach is to adhesively bond

\footnotetext{
a e-mail: noah.ledford@emi.fraunhofer.de

b e-mail: hanna.paul@emi.fraunhofer.de

c e-mail: georg.ganzenmueller@emi.fraunhofer.de

d e-mail: michael.may@emi.fraunhofer.de

e e-mail: M.Hoefemann@sz.szmf .de

f e-mail: M.Otto@sz.szmf.de

g e-mail: nikica.petrinic@emi.fraunhofer.de
}

threaded metalic endcaps on both ends of the specimen and subsequently screw the specimen and the endcaps into the bars. If the adhesion between end caps and specimen is not sufficient, additional clamping mechanisms can be used [3]. Another way is to use specimens with holes and screws to fix them in the bar [4]. It also possible to use an interlocking geometry and directly insert the tapered specimen into the bar [5]. In the work presented here, various ways of mounting flat specimens into the bars are investigated. Special attention is given to the influence of different mounting methods upon the stress waves measured using strain gauges attached to the loading bars away from the specimen, in order to assess the impedance of proposed mounting methods.

In the design of specimen geometry several parameters play key roles. The most important are the specimen shape, the length and the specimen cross section areas as well as the way of the tapering of the specimen, i.e. the transition from the gauge section to the shoulders. The length of the specimen influences the strain rate. The cross section area of the specimen is directly related to the force required to deform and break the material. In addition a reduction of cross section area at the center of the specimen is necessary to realize failure of the specimen away from the gripping zone. However the reduction of width is limited and dependent on the type of material tested. With a poor taper on the specimen, failure initiates at the end of the transition due to the stress concentration rather than in the middle of the parallel section, thus complicating the analysis and possibly invalidating the test.

\section{Experimental setup}

\subsection{The Split Hopkinson Tension bar (SHTB)}

To investigate the effects of the geometry and mounting options on the test results a Split Hopkinson Tension bar based on the design proposed by Gerlach [2] is used.

This is an Open Access article distributed under the terms of the Creative Commons Attribution License 4.0, which permits unrestricted use, distribution, and reproduction in any medium, provided the original work is properly cited. 


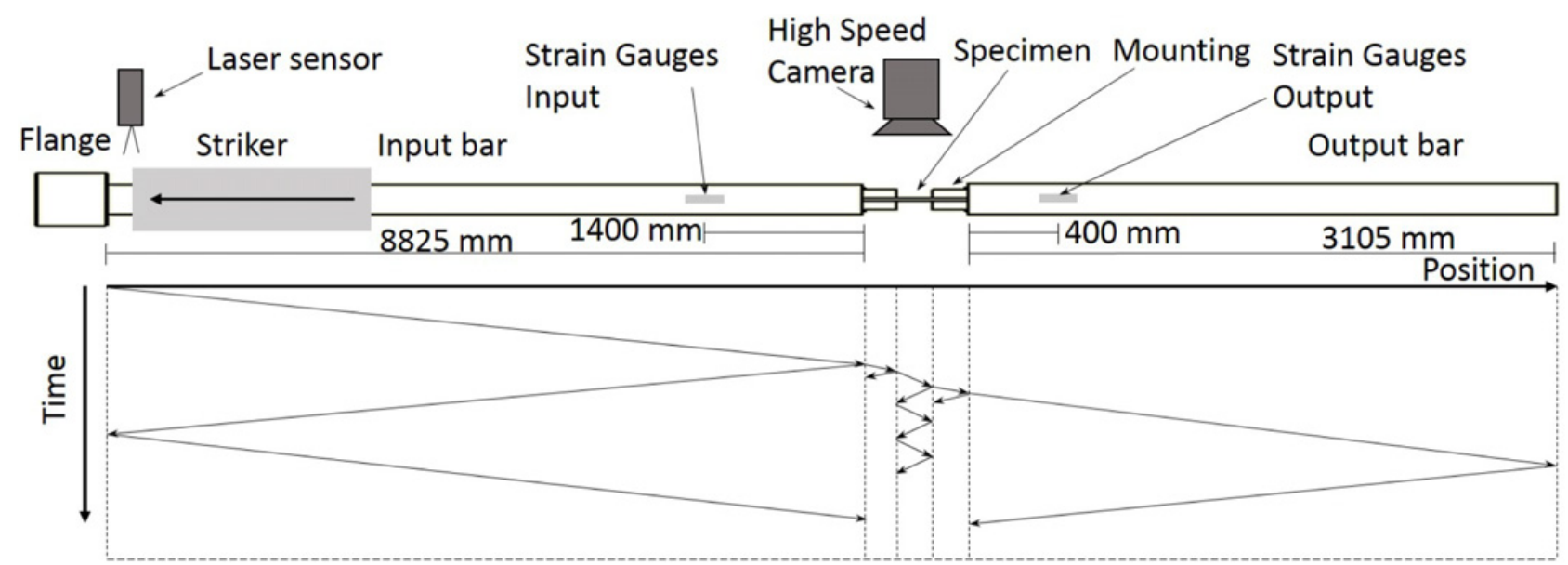

Figure 1. Schematic and Lagrange diagram of SHTB system.

Table 1. Mounting method qualitative comparison (++: better, +: good, -: not good, - -: worse).

\begin{tabular}{llllll}
\hline Criteria & Adhesively bonded & Dowel-pin & Form-fit & Clamped-inertia & Clamped-friction \\
\hline Stress Concentration & ++ & - & - & - & - \\
Additional mass & ++ & ++ & + & - & - \\
Impedance difference & + & + & - & - & - \\
Wave symmetry & ++ & + & - & - & - \\
Thermal limit & - & + & + & + & ++ \\
Specimen alignment & - & + & + & - & - \\
Through put Speed & -- & + & ++ & + & - \\
Cost & - & + & + & + & + \\
\hline
\end{tabular}

Figure 1 show a schematic of the SHTB. The Striker is accelerated by a pressurized air system and impacts the transfer flange on the far left. This imparts a tension wave into the input bar which travels through the bar towards the specimen. The tension wave is transferred to the specimen after passing through the threaded interface, between the input bar and the mounting hardware. The stress of the specimen is determined using the strain gauges on the output bar. To get the force transferred to the output bar by the specimen a calibration for the bar was performed. The displacement of the specimen is directly observed using a high speed camera and then the strain computed using Digital Image Correlation DIC, since better results can be achieved if strain is measured locally [6]. To measure the strain in the specimen a Vision Research Phantom 1610 high speed camera was used. These photographs were then imported into Gom ARAMIS software and the displacements and strains computed.

\subsection{Specimen mounting}

Various mounting method have been used for flat specimens: adhesively bonded end caps [3], form-fit grips [5], bolted (dowel-pin) [4] specimen into bars and clamping the specimen. Clamping can be based on the inertia of the clamps at dynamic experiments or based on mechanical force and friction. To compare the general aspects of the different methods Table 1 has been included to give an overview of the trade-offs involved in the different designs. The trade-offs below are compared where a plus sign indicates a positive relative comparison with the other methods and a negative sign indicates a lower score. The categories are roughly ordered based on importance but that will naturally depend on the project at hand. The authors recognize that the Table 1 is a subjective evaluation but feel it helps identifying the strengths of the different methods. The research in this paper has been conducted in order to help quantify these distinctions.

A short description of the terms above will help clarify the thinking behind the table. Stress concentration denotes the departure from uniform stress distribution across any cross section affected by specimen mounting and specimen geometry; this will have an effect on the stress state of the sample and will often cause the failure outside of the gauge section. Additional mass is a negative factor for mounting specimen. It is ideal to minimize this term having to accelerate more mass will lower the loading rate of the specimen. Impedance difference is the change in impedance between the bar and the mounting hardware. With a large difference there will be more reflections in the hardware which will affect the measured stress signal in the output bar. By wave symmetry the loading path is considered and how symmetric the transition from the round bar to the flat specimen is. The thermal refers to the ability to use the mounting methods at temperatures significantly below or above the room temperature. Specimen alignment is how difficult it is to center the specimen in the mounting hardware. Throughput speed considers how quickly can the specimen be prepared and exchanged to conduct multiple tests. And cost considers the cost of the hardware to conduct a test using the mounting method. 

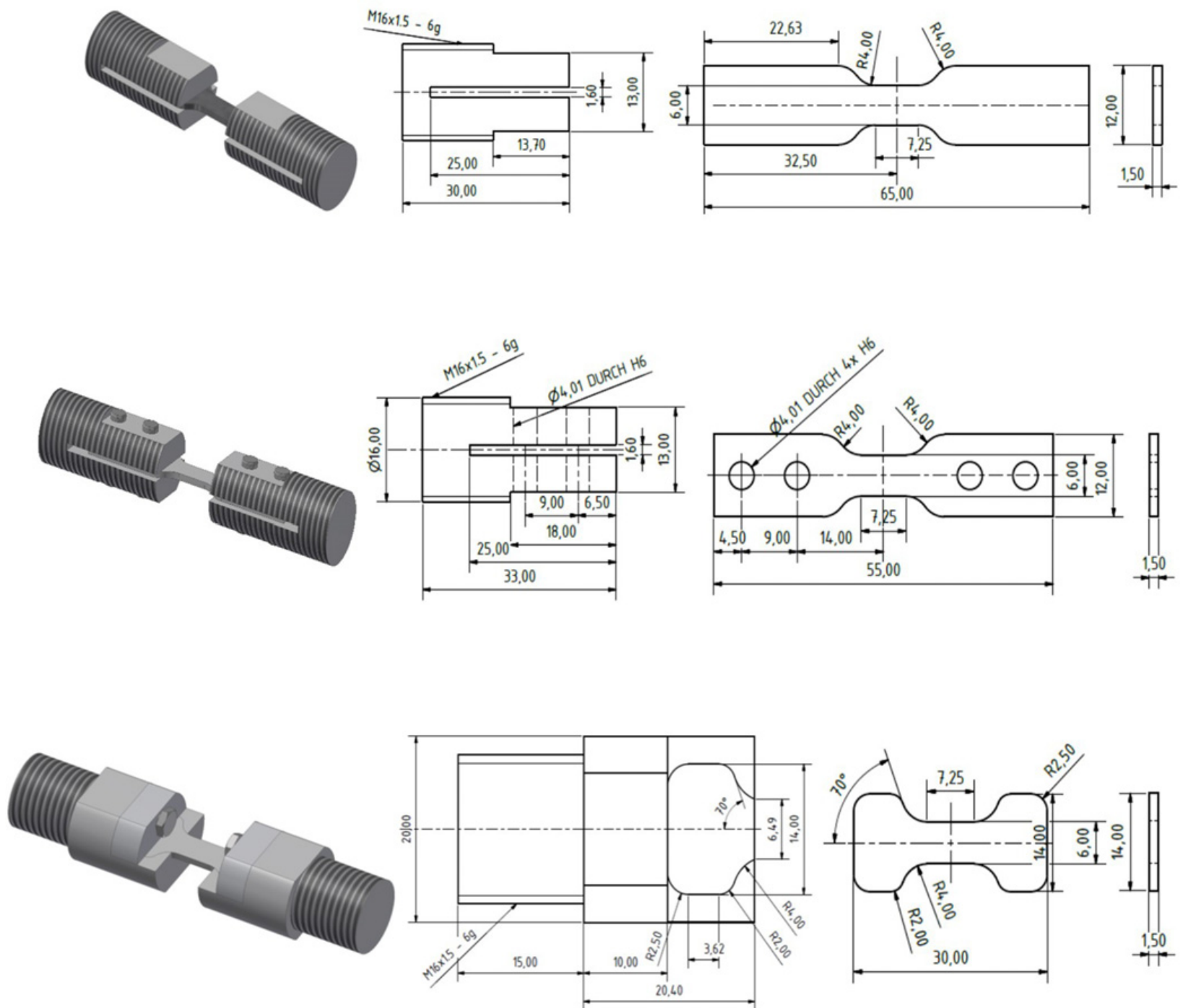

Figure 2. Different mounting methods: adhesively bonded mounting hardware (top), dowel-pin mounting hardware (middle), form-fit mounting hardware (bottom).

Since only room temperature tests were planned for the present project the adhesively bonded, dowel-pin and form-fit mounting were chosen for the investigations on the mounting methods.

For adhesively bonded specimens the mounting hardware consists of a threaded rod with a slit cut into the center where the specimen is adhesively bonded as shown in Fig. 2 (top). The adhesive used for the mounting of the specimen is LOCTITE EA 9514 which has a nominal shear strength of $50 \mathrm{MPa}$ with a cure time of an hour at $150^{\circ} \mathrm{C}$. The shear strength was used to determine the required surface area of the sample and consequently the length of the adhesively bonded mounting hardware. The amount of vertical space for the slit, $0.5 \mathrm{~mm}$ on both sides, was determined by the available tooling size and was found to be sufficient for a strong hold. The flat surfaces on the ends of the threaded rod are to allow a wrench to torque the mounting hardware into the end of the bars.

Dowel-pin mounting is using two dowels on each end of the specimen to attach it to the mounting hardware as shown in Fig. 2 (middle). Here the load is transferred through the dowel pins into the specimen. There is a stress concentration created on the pins. The design of the specimen needs to account for this and ensure that there is enough material at the connection to allow the specimen to break in the middle of the specimen. The tolerance on the dowel pin holes was selected allowing removal of the pins after a test so that the mounting hard ware could be reused.

The form-fit mounting option consists of a precisely cut specimen and mounting attachment that transfers the load on the tapered section of the specimen. This can be seen in Fig. 2 (bottom). In the form-fit mounting the specimen is placed into the mounting and the tension load is transferred through the tapered sides of the specimen and the mounting hardware. This will create a stress concentration and the geometry of the design is critical for successful use [5]. Finite element simulations were conducted to optimize the design to find an angle and end width that allows the stress to be spread in the ends of the specimen allowing for the failure to occur in the gauge section. A top is included on the hardware to ensure that 


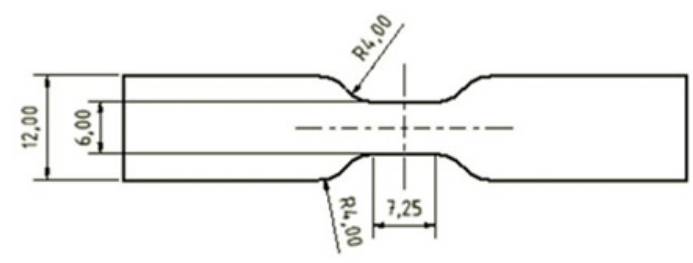

R 4

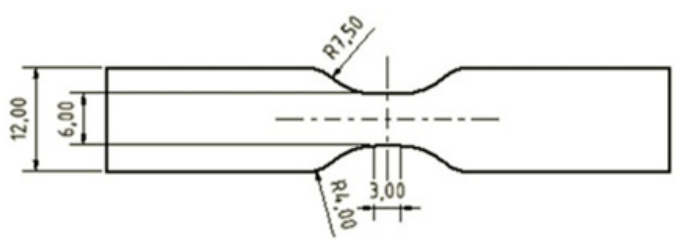

R 7.5

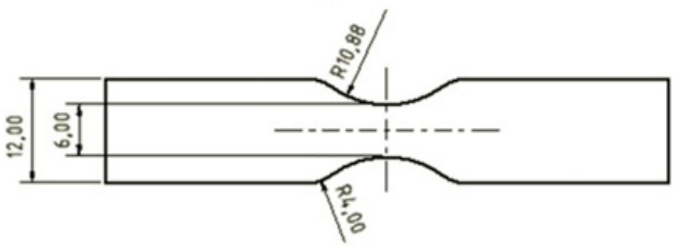

R 10

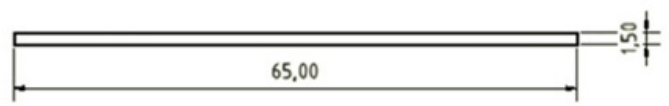

Figure 3. Dogbone specimen geometries.

the specimen does not come out of the pocket during the test possibly causing injury or damaging the camera.

For all design options, specimens with the same gauge cross section area and length of specimens as well as the same initial radius for the tapering were used. They were all tested using the same method and striker speed.

\subsection{Material and specimen geometry}

To ensure that the specimen breaks in the middle of the specimen dogbone specimen are used where the sides of the center section are tapered to a narrower width than the initial section that is secured in the specimen mounting. This tapering leads to a stress concentration in the specimen. To observe the effects of this stress concentration three geometries were selected and can be seen in Fig. 3. These specimen held constant the open length between the mounting connections and varied the radius of the transition from the initial width of $12 \mathrm{~mm}$ to the gauge width of $6 \mathrm{~mm}$. The radii chosen are a sharp transition of $4 \mathrm{~mm}$ radius and parallel gauge length of $7.25 \mathrm{~mm}$, a middle value of $7.5 \mathrm{~mm}$ with a parallel length of $3 \mathrm{~mm}$, and a large radius of $10.88 \mathrm{~mm}$ where there is no parallel gauge length. For the evaluation of the specimen geometry the adhesively bonded mounting was used. The material the specimen were made out of a high strength steel. The material is being developed by Salzgitter.

\section{Results}

\subsection{Mounting method}

Figure 4 shows the results of specimens tested with the different mounting methods. For all shown results the specimen geometry was R4 (see Fig. 3). There are ten results from tests with the adhesively bonded mounting and three from the form-fit and the dowel-pin mounting.

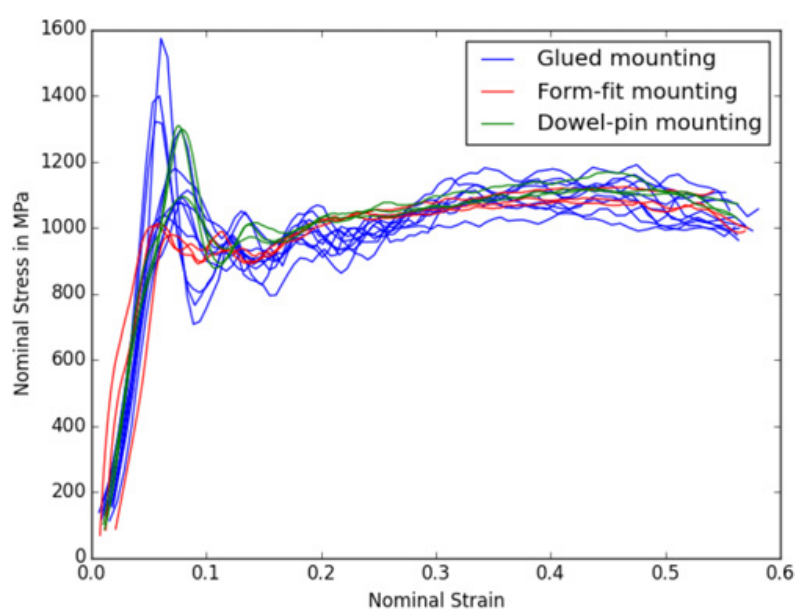

Figure 4. Nominal stress strain curves with different mounting hardware.

The results are shown as nominal stress versus nominal strain curves. This means that the stress is calculated using the initial cross section area of the specimen gauge section. The strains were measured using Digital Image Correlation. For the calculation of the strains displacements at two points with an initial distance of $7 \mathrm{~mm}$ were evaluated. With all mounting methods the same principal behavior is measured. The stresses and strains at the end of the tests are in the same range. There is a difference of the mounting methods at the beginning of the test. Each mounting method results in a different amount of oscillation. There is little oscillation for the form-fit mounting method. Both the dowel-pin and the adhesively bonded mounting produce results with significant oscillation.

It is obvious that the dowel-pin mounting results in oscillations, since the slit in the mounting is slightly bigger than the thickness of the specimen. In order to reduce the oscillations the slit needs to be reduced exactly to the specimen thickness. Since the specimen is firmly fixed for the adhesively bonded mounting the oscillations are not obvious in this case. One possibility is that the specimens are not positioned in the center of the endcaps (in hight and in line of the bars). This can result in lateral oscillations of the specimens which leads to the oscillations of the results. In the form fit mounting the correct positioning of the specimen is easily achieved.

The strain rate can be estimated with the striker velocity and the length of the parallel section of the specimen, since most of the elongation will happen in this area. The striker velocity was at $12 \mathrm{~m} / \mathrm{s}$. All specimens for the tests for the inverstigation of the mounting methods have a parallel section of $7.25 \mathrm{~mm}$. This results in a expected strain rate of $1655 \mathrm{~s}^{-1}$. Figure 5 shows the strain rate of the strains measured on the specimens. At all specimens the strain rate at the beginning and at the end of the test is at about $1200 \mathrm{~s}^{-1}$. During the test there is a drop of strain rate. One reason for the lower actual strain rate is that the parts of the specimens beyond the parallel section are loaded as well. Another reason is that there is deformation in the mounting. For the form-fit mounting the drop is much higher than for the other two 


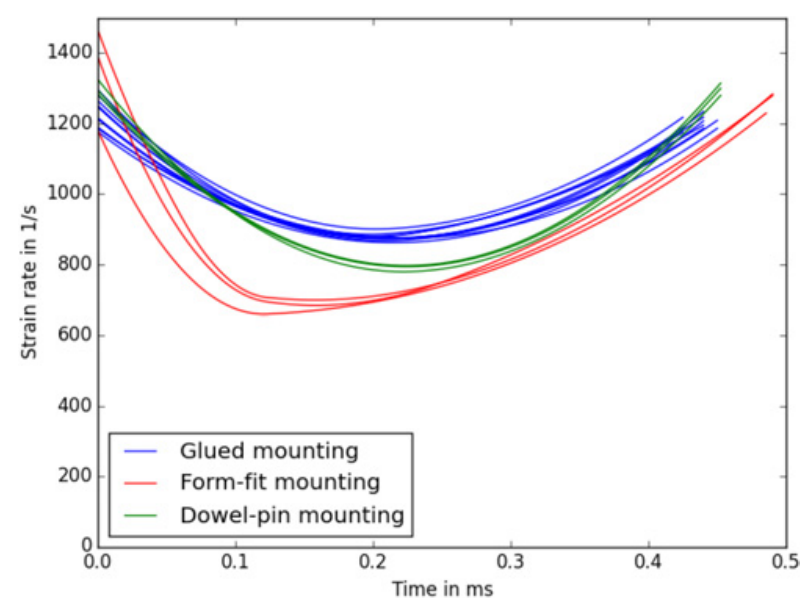

Figure 5. Strain rate with different mounting hardware.

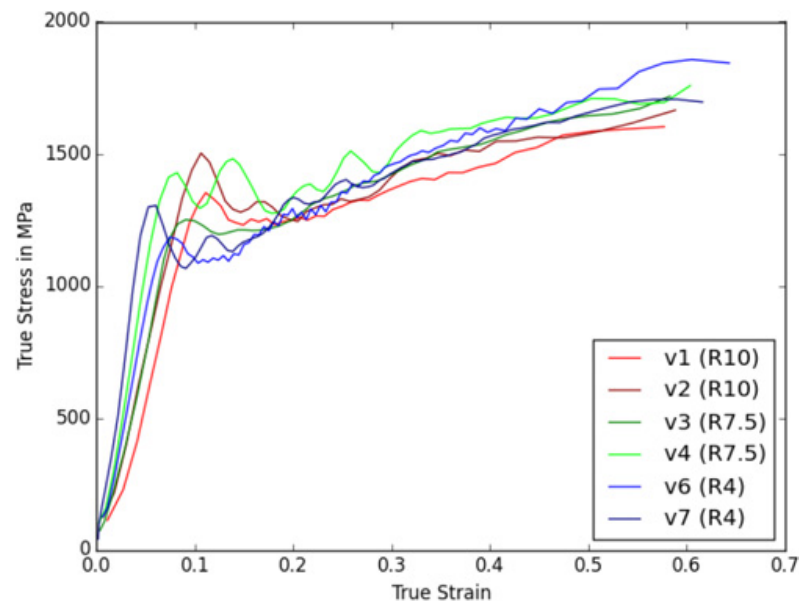

Figure 6. Local true stress strain curves for different specimen geometries.

mounting methods. There might be more deformation in the mounting than at the other mounting-methods.

\subsection{Specimen geometry}

Three different specimen geometries with different tapering were investigated (Fig. 3). Due to the chosen tapering, the length of the parallel section is different. For this reason the strains were evaluated as local true (logarithmic) strains in the fracture area. Additionally the strains perpendicular to the loading were used to calculate actual cross section area of the specimen and with that the true stresses. Figure 6 shows local true stress strain curves for the three tested specimen geometries. There is no significant influence of the specimen geometry on the results.

Figure 7 shows the local strain rates calculated from the local strains in the fracture area of the specimens. In contrast to the strain rates displayed in Fig. 5 which were calculated at the ends of the parallel section the local strain rates have a significant rise before failure. This can be explained with the localization of the material. There is a significant difference in strain rates for the different

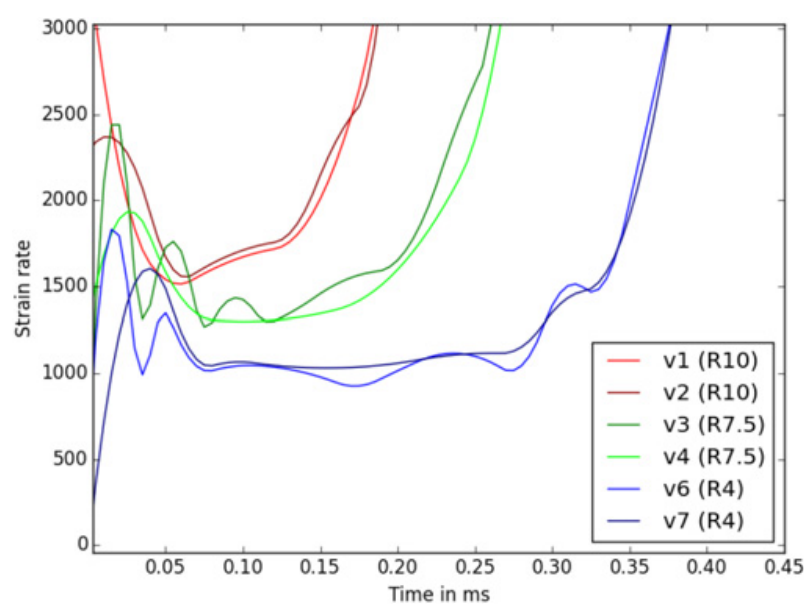

Figure 7. Local strain rates for different specimen geometries.

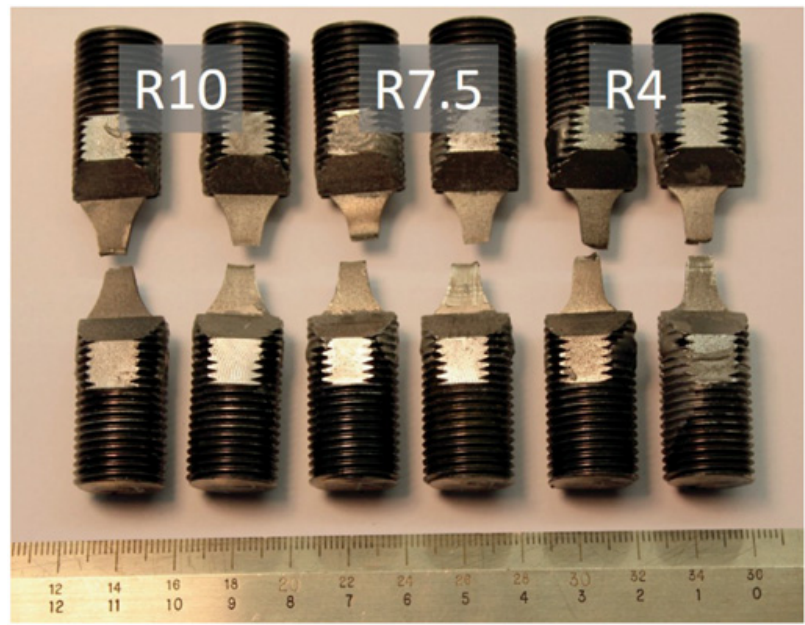

Figure 8. Investigation of influence of specimen geometry: Failed specimen.

specimen geometries. The specimen with the bigger radius (R10) has a higher localization of strain and with this a higher strain rate. Since the failure for all shown specimens was in the middle (see Fig. 8) the geometry with the smaller radius (R4) is favored.

\section{Conclusions}

In this work different mouting methods for Split Hopkinson Tension Bar Experiments (SHTB) were investigated. For the investigated sheet metal all three mounting methods (adhesively bonded, with dowel pin and with form-fit) give reproducible results. The adhesively bonded and the dowel-pinned hardware lead to oscillations at the beginning of the tests. The best results were gained with the form-fit mounting. The strain rate has a minimum in the middle of the test. The drop is higher for the form-fit mounting. Summing up all mounting methods are suitable for SHTB experiments. But since the form fit mounting is cost effective and leads to the best signal this mounting should be used for this kind of materials. 
In the second part three different specimen geometries were investigated. For this specific material all specimen geometries lead to a failure in the center and are therefore possible to use. For the specimen with the smallest radius and the longest parallel section the loading is less localized and therefore this geometry is favored.

It has to be highlighted that the results are valid only for this kind of material with a fairly high fracture strain. For materials such as carbon fiber composites with a linear elastic material behavior and projects with other constraints other mounting methods and specimen geometries might be appropriate. A possibility to gain better results with the adhesively bonded mounting method would be pulse shaping.

In order to get a better understanding on the influence of positioning and the mass of the mounting on the results it is intended to preform finite element simulations of the Split Hopkinson Tension Bar experiments.

\section{References}

[1] Chen W., Song B., Split Hopkinson (Kolsky) Bar Desing, Testing and Applications (Springer, New York Dordrecht Heidelberg London, 2011)

[2] Gerlach R., Kettenbeil C., Petrinic N., International Journal of Impact Egnineering 50, 63-67 (2012)

[3] Gerlach R., Siviour C.R., Wiegand J., Petrinic N., Composites Science and Technology 72, 397-411 (2012)

[4] Huh H., Kang W.J., Han S.S., Experimental Mechanics 42, 8-17 (2002)

[5] Owens A.T., Tippur H.V., Experimental Mechanics 49, 799-811 (2009)

[6] Schmidt T., Gilat A., Walker A., Seidt J., Tyson J., Proceedings of the Xth International Congress and Exposition, Society of Experimental Mechanics, Orlando (2008) 